\title{
Quadratic equations in Swedish textbooks for upper-secondary school
}

\author{
Wang Wei Sönnerhed \\ Department of Education, Communication and Learning at University of Gothenburg, Sweden
}

\begin{abstract}
This paper analyzes the content and tasks involving quadratic equations in eight mathematics textbooks published during the period 2000-2012 at the uppersecondary level in Sweden. The study applies the theoretical hypothetical learning trajectory (HLT) framework combining conceptual and procedural knowledge. The analysis includes horizontal and vertical dimensions within an HLT. The aim is to explore embedded HLTs and learning opportunities from both dimensions in these textbooks. A total of 250 examples and 1,068 tasks have been examined. Results show that all the textbooks contain algebra identities and four different methods for solving quadratic equations as well as their applications as a core hypothetical learning trajectory but differ in how an HLT starts and ends. Geometrical representations for some algebra identities and completing the square are widely used in both theoretical presentations and tasks, which implies that conceptual learning is encouraged among the Swedish textbooks. At the same time, procedural knowledge is still emphasized as a basic but important learning process.
\end{abstract}

ARTICLE DETAILS

LUMAT General Issue Vol 9 No 1 (2021), 518-545

Received 12 December 2020 Accepted 6 June 2021

Published 21 June 2021

Pages: 28

References: 34

Correspondence:

wei.sonnerhed@ped.gu.se

https://doi.org/10.31129/ LUMAT.9.1.1473

Keywords: Algebra, hypothetical learning trajectory, quadratic equations, textbook analysis, visual representations

\section{Introduction}

In the Swedish national mathematics curriculum (2011) for second-year uppersecondary schools, quadratic equations are among the central content. The same content is taught in many other countries in the world, although in different years of high school. Within the area of quadratic equations, students often study algebra concepts and identities, different solution methods, and applications of quadratic equations in problem-solving. Solving a quadratic equation using the $p q$ formula $^{1}$ is one of the most common solution methods in Swedish classrooms (Olteanu \& Holmqvist, 2012). Demanding prior knowledge of many algebra rules or identities, quadratic equations take up much knowledge space in the curriculum and form a complex teaching area. Therefore, it is important to present this new content comprehensively so that students will have opportunities to understand the abstract

${ }^{1}$ The $p q$ formula is a quadratic formula of the type $x_{1,2}=-\frac{p}{2} \pm \sqrt{\left(\frac{p}{2}\right)^{2}-q}$. It is used for solving quadratic equations of the type $x^{2}+p x+q=0$. 
content.

Different approaches for students to solve quadratic equations have been studied since 2000; for example, factoring quadratics (e.g., Bossé \& Nandakumar, 2005), completing the square with geometry representations (e.g., Allaire \& Bradley, 2001; Fachrudin et al., 2014), and using the $p q$ formula (e.g., Olteanu \& Holmqvist, 2012). Learning and understanding quadratic equations and their solution methods have also been studied; for example, students' understanding of quadratic equations (e.g., Vaiyavutjamai \& Clements, 2006), and students' understanding of factoring quadratic equations and their difficulties (e.g., Didis \& Erbas, 2015). Sharing the current study's focus, a number of recent studies directly relate to the analysis of different solution methods, for instance, a Polish study on reviewing traditional solution methods in the Polish curriculum and textbooks (Pieronkiewicz \& Tanton, 2019). The Polish study demonstrates applications of Viete's formula ${ }^{2}$ and the $\mathrm{AC}$ method 3 , which are methods of factoring quadratic trinomials in solving quadratic equations for two types of quadratic equations: $x^{2}+p x+q=0$ and $a x^{2}+b x+c=0$. The study also showed that the application of area models, which is based on geometrically using the combinations of rectangles and squares, to solve quadratic equations through completing the square is found in the textbooks. This approach originates from the history of the stages of algebra (e.g., Katz \& Barton, 2007). Unlike using traditional approaches for solving quadratic equations, an American study suggests using different graphs of quadratic functions drawn with the program GeoGebra to help students understand the quadratic formula through working with the symmetry of a graph (Edwards \& Chelst, 2019). Some studies are textbook analyses, comparing the content of quadratic equations in textbooks from different countries (Hong \& Choi, 2014; Saglam \& Alacaci, 2012; Winsløw, 2004). Most of the studies mentioned above focus on one or a few solution methods within areas of learning or teaching quadratic equations or analyzing textbooks' content. However, studying a whole learning process within the content involving both algebra rules as prior knowledge of and solution methods for quadratic equations, there seems to be a lack of attention to

\footnotetext{
${ }^{2}$ Viete's formula is $r_{1} \cdot r_{2}=q ; r_{1}+r_{2}=-p$ if $r_{1}$ and $r_{2}$ are the roots to the equation type $x^{2}+p x+q=0$. This type of equation can be solved by finding a pair of integers for which the product is $q$ and the sum is $-p$. The integers found are the roots to the equation.

${ }^{3}$ The AC method is based on the idea of Viete's formula and is used for equations of the type $a x^{2}+b x+c=0$. It contains four steps: find $a c$; find the factors of $a c$ which add to $b$; if these factors are $p$ and $q$, replace $b x$ with $p x+q x$; complete the factorization (Pieronkiewicz \& Tanton, 2019, p. 109).
} 
quadratic equations in mathematics education.

Regarding how quadratic equations and the related content are presented in detail as a whole learning process, analyzing mathematics textbooks is an efficient way to examine what is offered to help students learn this abstract content. Textbooks contain not only subject-content knowledge but also pedagogical intentions (Pepin et al., 2001). Textbooks are widely used in mathematics classrooms in Sweden (Jablonka \& Johansson, 2010; Madej, 2021). Mathematics teaching in Swedish classrooms is often based on textbooks (Madej, 2021). Mathematics teachers plan and prepare their lessons mainly by use of textbooks, and $45 \%$ of the teachers use the textbooks as exercises books (Lepik, et al., 2015). Students spend a great amount of time doing exercises from textbooks and the mathematics knowledge teachers present in the classroom mainly stems from the textbooks they use (Bergwall \& Hemmi, 2017; Johansson, 2006). As intended, implemented and enacted curriculum material, textbooks play an important role in mathematics education (Valverde, et al., 2002). They contain embedded pedagogy by reflecting content in a certain way to suggest appropriate sequences of the content and pedagogical situations where activities, explanations, examples and exercises are selected to play particular roles; these roles are fixed and unchanging in textbooks (ibid, p. 12). Cognitive requirements on mathematics tasks provide different types of tasks and offer students varied opportunities in learning mathematics (Gracin, 2018). Teaching, learning and using artifacts converge in a textbook and make it multifunction in transferring subject knowledge. Considering embedded pedagogy and a wide range of content on algebra elements and quadratic equations in sequences, the author of this article chooses to analyze mathematics textbooks instead of studying classroom interactions on students learning quadratic equations within a limited area. The study's aim is to explore an embedded hypothetical learning trajectory (HLT) for learning quadratic equations from algebra rules to solving and applying quadratic equations to a wide extent by analyzing the related content, including tasks in Swedish mathematics textbooks. The research questions for this study are:

1. What embedded hypothetical learning trajectories regarding quadratic equations can be identified in Swedish textbooks for helping students understand abstract algebra concepts and procedures?

2. What learning opportunities regarding quadratic equations within an embedded HLT are offered by tasks in Swedish textbooks? 


\section{Previous studies on mathematics textbooks regarding algebra}

The current study analyzes mathematics content in textbooks as subject matter knowledge, which in the textbook analysis is defined as a product-oriented approach (Johnsen, 1993). Some Nordic studies have involved analyzing mathematics textbooks in relation to the curriculum and subject of school algebra. Based on the framework of algebra big ideas ${ }^{4}$, Hemmi et al. (2018) exam how algebra is presented in Swedish school curricula and textbooks as well as teachers' discourses for grades 19 from the diachronic and synchronic perspectives in order to characterize Swedish school algebra. It is found that EEEI is the most represented category in the textbooks for grades 1-3 and even 4-6; FT and VAR are also well represented, whereas GA is the least represented category in the textbooks. The focus study by Hemmi et al. (2019) has been carried out to analyze introductions of early school algebra particularly related to EEEI and GA in textbooks for grades 1-3 in Estonia, Finland and Sweden (Hemmi et al., 2019). The focus study finds that inverse properties are used in textbooks from the three countries. Creating letter expressions and equations are found in Estonian textbooks. With the same framework, the related study on identifying algebra thinking in Swedish primary textbooks and curriculum (Madej, 2021) shows that EEEI is the main algebra idea in the Swedish context but not GA. Palm Kaplan (2019) analyzes six mathematics textbooks for Swedish lower-secondary schools published in 1995-2015 in order to understand algebra characteristics in terms of school algebra discourses and algebraic activities. The results show that five algebra discourses 5 are identified in the textbooks. An early study identified school algebra according to three periods ${ }^{6}$ in Swedish upper-secondary textbooks for the years 1960-200o by Jakobsson-Åhl (2006) with the approach of phenomenography and hermeneutics.

To sum up, school algebra in analyzed Swedish textbooks for primary and lowersecondary schools mainly involves categories of equivalence, expressions, equations, and inequalities (EEEI); function thinking (FT) and variables (VAR) have also been found. Algebra as generalized arithmetic (GA) is not found in the Swedish textbooks

\footnotetext{
${ }^{4}$ Algebra big ideas as an analytical framework used by Blanton et al (2015) in Hemmi et al. (2018) for studying students' algebraic thinking. They refer to 1) equivalence, expressions, equations and inequalities (EEEI); 2) generalized arithmetic (GA); 3) functional thinking (FT); 4) variable (VAR).

${ }^{5}$ Five algebra discourses are symbolic, arithmetical, geometrical, (un)realistic and scientific.

${ }^{6}$ Three algebra periods are pre-New Math, New Math and post-New Math.
} 
for years 1-9. School algebra in the analyzed textbooks at Swedish upper-secondary level has been developed from the focus of algebra manipulation to algebra application in real-world problems. Despite the various studies relating to analyzing the contents of algebra in textbooks, none of them directly focuses on quadratic equations.

However, three international studies (Hong \& Choi, 2014; Saglam \& Alacaci, 2012; Winsløw, 2004) look at quadratic equations in textbook analyses. Among these studies, some textbooks regard polynomials or binomials or geometrical representations of quadratics as prior knowledge before quadratic equations (Saglam \& Alacaci, 2012). Introducing quadratic equations with a real-world problem (Hong \& Choi, 2014; Winsløw, 2004) or by reviewing linear equations (Hong \& Choi, 2014) or directly presenting the quadratic formula after the formal definition of quadratic equations (Winsløw, 2004) are different ways to approach quadratic equations in analyzed textbooks. Solving quadratic equations with factoring and completing the square illustrated with geometrical representations are found as common solution methods (Hong \& Choi, 2014; Winsløw, 2004), while some quadratic equations are solved with the square root method or graphical approach (Winsløw, 2004). Different pedagogical intentions have been explored in these studies. For example, encouraging students to reason and explain the concepts, and to use the mathematical thinking process rather than algorithms (Hong \& Choi, 2014); or emphasizing the procedural practice of using the formula (Winsløw, 2004). Among these three studies, there is an apparent lack of information in their results as to exactly which topics and what types of tasks have been examined, and where they start and end, as well as how the content is organized in order to develop abstract algebraic thinking.

To benefit research on school algebra in Swedish textbooks at upper-secondary school level, the current study analyzes how various algebra content regarding quadratic equations, including theoretical presentations with given examples and provided tasks, are connected and developed as a whole hypothetical learning trajectory utilizing the theoretical framework of hypothetical learning trajectory (HLT) (Simon, 1995). 


\section{Analytical approach}

\subsection{General framework}

The general analytical framework used in this study is based on the concept of HLT (Simon, 1995; 2014). An HLT (Simon, 1995) "is made up of three components: the learning goal that defines the direction, the learning activities, and the hypothetical learning process - a prediction of how the students thinking and understanding will evolve in the context of the learning activities" (p.136). "The latter two parts are interdependent" (Simon et al., 2018, p. 102). It is a theoretical model for the design of mathematics instruction (Simon, 2014). The central pedagogy of HLT is mathematics teaching for understanding (Simon \& Tzur, 2004). Its aim is to develop students' mathematics thinking within a designed conceptual learning progression through different sequences of tasks. HLT "refers to the teacher's prediction as to the path by which learning might proceed. It is hypothetical because the actual learning trajectory is not knowable in advance. It characterizes an expected tendency" (Simon, 1995, p. 135).

Simon and Tzur (2004) emphasize that tasks selected for learning activities play an important role in providing effective mathematics instructions, and cognitively demanding tasks can develop students' cognitive abilities and mathematics thinking. Compared to routine tasks, which are applied as procedural practice, cognitively demanding tasks can offer students opportunities to learn mathematics concepts.

The analyses of mathematics examples and tasks in this study applied the concepts of conceptual and procedural knowledge of mathematics (Hiebert and Lefevre, 1986). Conceptual knowledge is characterized as knowledge that is rich in relationships and can be thought of as a connected web of knowledge (ibid, p. 3). Conceptual knowledge grows through the construction of relationships between existing knowledge and new information. It is labeled as meaningful learning. While "procedural knowledge is made up of two distinct parts. One part is composed of the formal language or symbol representation system of mathematics. The other part consists of algorithms or rules, for completing mathematical tasks" (ibid, p. 6).

Different cognitive demands on mathematics tasks provide opportunities for students to develop procedural and conceptual knowledge. Gracin (2018) interprets cognitive demands on mathematics tasks based on features of rich tasks, low- and high-level tasks, and mathematical competencies. Rich mathematics tasks have high cognitive demands: 1) memorization, 2) procedures without connections, 3) 
procedures with connections while at the same time developing deeper levels of understanding of mathematical concepts and ideas, and 4) doing mathematics requiring complex and non-algorithmic thinking with great cognitive effort. Low-level tasks contain the first two features, while high-level tasks are based on the last two features and often require students to understand, interpret, apply mathematics knowledge and skills from different sources to accomplish work. A balanced curriculum requires both low- and high-level tasks with a full range of problem types. High cognitive demands in mathematics competencies (Niss, 2015) entail mathematical thinking, problem handling, modeling, reasoning, representation, symbol and formalism, communication, and aids and tools.

In the current study, the HLT is used in such a manner that the author explores an embedded HLT concerning quadratic equations in eight textbooks through identifying three components: the learning sub-goals and final goals; the intended hypothetical learning processes; and the learning activities, as provided learning tasks in the respective related topics. As the overall learning goals are the same in all the textbooks - that is, how to solve and use quadratic equations - this analysis focuses on exploring the embedded learning process and analyzing related learning tasks within and between learning sub-goals. The learning sub-goals are directedly related to the topics before the final goals of solving and applying quadratic equations are addressed. The HLT is applied at two levels: a major level in a horizontal dimension, there theoretical presentations including examples within every topic from basic algebra concepts and rules to quadratic equations, are examined; a minor level in a vertical dimension, there provided tasks under every topic, are analyzed (Figure 1).

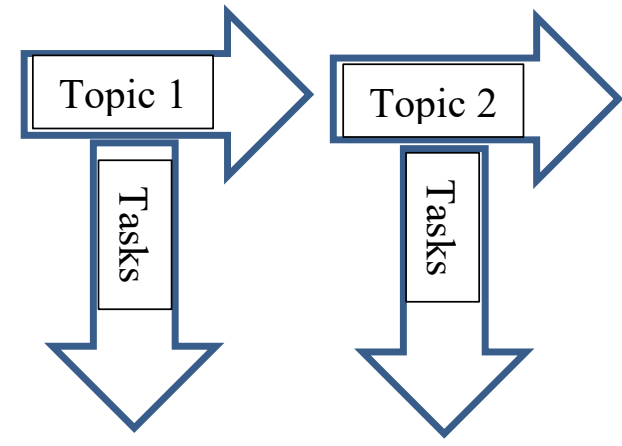

Figure 1. The whole HLT at two levels consists of a number of topics as content in a horizontal dimension. Every topic provides a group of tasks in a vertical dimension.

At the major level, an HLT is interpreted through exploring how every single topic is presented and exemplified so that students can understand an algebra concept or 
rule, later how the single topics as sub-goals connect each other to reach the final goal of solving and using quadratic equations in a textbook so that intended mathematics thinking develops from basic stages to abstract level as a hierarchical order. The horizontal HLT is examined as potential instructional material provided for teaching.

At the minor level, analyses of learning tasks within a single topic are carried out through applying analytical categories: procedural and conceptual knowledge; applications; and the framework of HLT. Hiebert and Lefevre (1986) expressed that tasks aimed at training students in mathematical procedures are applied to foster their procedural knowledge regarding rules and procedures for solving mathematics problems; tasks focusing on training in mathematics concepts are intended to foster students' conceptual knowledge, which involves a deeper understanding of mathematical relationships. In line with the category of conceptual knowledge, tasks focusing on applying recently learned mathematics concepts, rules, or solution methods for solving real-world mathematics problems are identified as applicational tasks which require comprehension, interpretation, and application of mathematical knowledge and skills (Gracin, 2018). In a sequence of tasks, the embedded HLT is identified by determining how the provided tasks are arranged and developed from basic computation procedures to abstract mathematics generalization in a hierarchical order.

The analyses of tasks intend to explore what learning opportunities are provided for students to learn basic algebra concepts and rules; and solving and using quadratic equations in a textbook from a learning perspective.

\subsubsection{Analytical criteria in the vertical dimension}

In detail, mathematics tasks are categorized in this study as such:

1. Tasks require students to simplify an algebra expression with algebra identities or rules; to factorize an algebra or quadratic expression or equation; to solve a linear or quadratic equation; to draw the graph of a quadratic function; and to find solutions for quadratic equations using a graphical calculator. These are categorized as procedural tasks. The characters of this type of task involve the following: formal mathematical symbols, algorithms and rules (Hiebert and Lefevre, 1986); the solution procedures or algorithms have been presented in previous theoretical presentations; the procedures can be found in textbooks and imitated; and they are routine tasks (Brändström, 2005). 
2. Unlike procedural tasks, tasks requiring students to do mathematics through investigating, generalizing, reasoning, assessing, proving, and so on are categorized as conceptual tasks. They are carried out by connecting task information to existing knowledge (Hiebert and Lefevre, 1986). The following are different types of requirements for conceptual tasks in the eight textbooks:

- Requiring students to find the missing parameter (an unknown, a coefficient, or a constant) in a given algebra identity or an equation; or find the relationship among a coefficient, a constant, and roots of a quadratic equation.

- Requiring students to prove or explain algebra identities or rules using geometrical representations or in words.

- Requiring students to evaluate or assess whether a mathematics statement or calculation procedures or solutions to quadratic equations are correct, and if not, to reason why it is wrong and give a correct answer.

- Requiring students to set up an algebra expression or quadratic equation according to given statements, a word problem, or a visual representation through interpreting given contexts and applying algebra ideas and symbols to solve it.

3. Tasks asking students to solve a word problem in the application of an algebra expression or a quadratic equation are called applicational tasks in this study. Word problems are often constructed by relating to living world subjects: economy, geometry, physics, and arithmetic. In this study, such problems are called real-world problems; while they are not authentic real-world problems, they are intended to be familiar from students' daily life. Most of them can be solved with the help of the given information in a textbook. They require students to set up a mathematical model, such as an algebra expression or a quadratic equation, after interpreting the given text. However, a few of them require students to exercise high mathematical thinking, which means that the information needed to solve a task is not fully provided in the textbook (Palm, Boesen \& Lithner, 2011).

4. A sequence of tasks requires students to first calculate a certain type of tasks and discover the patterns of calculation, then generalize or prove them with algebra expressions or rules, finally prove, apply, or develop the rules. The goal is to understand abstract mathematics through a learning progression in a hierarchical order. The sequence of these steps consists of both procedural and 
conceptual knowledge and is usually constructed from simple to abstract steps. This category is a level above the first three categories since it covers all of them. This type of tasks is called HLT-tasks.

Briefly, the criteria of four categories of tasks are mainly based on procedural and conceptual knowledge (Hiebert \& Lefevre, 1986) and the HLT framework (Simon \& Tzur, 2004). At the same time, the cognitive requirements for Categories 2-4 are in line with the cognitive requirements for high-level tasks (Gracin, 2018) and mathematics competencies (Niss, 2015).

\section{Method}

The analyzed textbooks are eight Swedish mathematics textbooks used within the national program of Natural Science (NV) at the upper-secondary level (Appendix A). The textbooks were, and are, frequently used just before and after the establishment of the new national curriculum (Skolverket, 2011), according to information from the library of the Swedish National Resource Center for Mathematics. Half of the books (Matematik 5000 2c, Matematik 2c, Exponent 2c, Origo 2c) were published in 20112012, and have content on quadratic equations with complex numbers and solving a root equation, as well as analyzing a discriminant of a quadratic equation, which follows the requirement of the new curriculum. The other half (Delta NT/a+b,Nya Delta A och B, Exponent B Röd, Matematik 4Ooo Blå), published before 2011, contain quadratic equations dealing with real numbers only.

The analyses covered all the related topics and their respective tasks in every book. They were carried out in both horizontal and vertical dimensions in order to find a whole embedded HLT. The horizontal dimension analysis started with the topics of polynomials, algebra identities, or simplifying linear expressions, and ended with applications of quadratic equations. The vertical dimension analysis focused on tasks provided within every topic. A total of 273 pages were analyzed, covering 250 theoretical examples and 1,068 tasks.

For the horizontal dimension, the analysis was carried out in two steps (Steps 1 and 2), and for the vertical dimension, the analysis included one step (Step 3). The last analysis (Step 4) generalized both dimensions.

To find a whole HLT concerning the related topics with theoretical presentations and illustration examples in each book, the analysis of Steps 1 and 2 answered the following questions: 
1. How does the textbook prepare students for learning quadratic equations? This is determined by how early the first topic appears.

2. Which topics or sub-goals of learning pre-knowledge to quadratic equations and quadratic equations are there in a whole HLT?

3. How is the topic of quadratic equations introduced?

4. How does the HLT end?

5. How are all the topics organized?

6. In which order are the topics presented?

7. What examples and related visual representations are applied in order to present algebra concepts, rules, and solution methods?

In the analysis of Step 1, all the topics concerning concepts, rules, methods, and examples including visual representations in every book were written briefly and listed according to the following categories: basic algebra concepts named as preknowledge, for example the concept of polynomials (Questions 1); topics before quadratic equations such as algebra identities and rules as pre-knowledge (Question 2); introduction and presentation of quadratic equations (Question 3); solution methods or application of quadratic equations as a final goal (Question 4); the order and organization of all the topics (Questions 5,6); all examples including related visual representations within each topic (Question 7). The aim of Step 1 was to find an overall organization of a whole HLT in each book.

Step 2 aimed to summarize and compare all the HLTs of the eight books. All the HLTs were then generalized in five learning processes at most depending on different final learning goals according to the results of Step 1: 1) basic algebra concepts as preknowledge; 2) algebra properties and identities as pre-knowledge; 3) quadratic equations and their solution methods; 4) application of quadratic equations or finding the relationship 7 between parameters and roots of quadratic equations or discriminants; and 5) other content. The analyses of Steps 1 and 2 aimed to answer the first research question.

Step 3 was a vertical dimension analysis to answer the second research question. In this analysis, all the tasks provided for every topic in each book were categorized as types according to the four analytical criteria in Section 3.1.1: procedural, conceptual,

\footnotetext{
${ }^{7}$ Relationship between parameters and roots of a quadratic equation refers to the relationship between a constant $q$, a coefficient $p$ and roots $x_{1}$ and $x_{2}$ in the common quadratic equation $x^{2}+p x+q=0$. In Swedish textbooks, this relationship can be expressed as $p=-\left(x_{1}+x_{2}\right) ; q=x_{1} \cdot x_{2}$.
} 
applicational, and HLT-tasks. An example of a sequence of HLT-tasks is that students are first required to multiply two same binomials such as $(x+1) \cdot(x+1)$ and to do five similar tasks; second to discover a pattern of the results; third to square some new binomials according to the pattern at Step 2; fourth to generalize formulas of $(a \pm b)^{2}$ which are perfect square rules over addition and subtraction; at last discover a new rule $(a+b) \cdot(a-b)$ (Alfredsson et al., 2011, p. 75). In this example, the HLT of learning and understanding perfect square rules (as a learning goal) was explored through the development of cognitive requirements of the five tasks (as learning activities): from a simple procedure as multiplying, then to discovering a common pattern of the results, later to generalizing the rules, at last to challenging another new rule (as a learning process). Notice that procedural and conceptual types of tasks are included in this sequence.

The organization of the analysis in Step 3 entailed listing all the related topics of each book and all tasks provided within each topic according to the four types. Tasks were analyzed by examining the instructions for each task in order to translate its cognitive requirement; noting and categorizing whether it was a procedural, conceptual, applicational type of task, or a sequence of HLT-tasks; and then further analyzing in detail each type. For example, the detailed analysis of conceptual tasks was carried out by first briefly listing the cognitive demands in each book, then comparing and noting the common terms among the eight books, and finally categorizing them into different types. Its aim was to determine how every textbook offers opportunities to deeply understand algebraic thinking. The number of tasks in each type was counted in every book. Solving and computing tasks were carried out when it was difficult to interpret them directly.

After the analyses in the three steps above, and to confirm the answers to the two research questions, the analysis in Step 4, as a summary, compiled the previous analyses of both the horizontal and vertical dimensions. In the horizontal dimension, the HLTs derived from the analysis of Step 2 made up the main structure horizontally, consisting of all the analyzed topics. In the vertical dimension, the total number of tasks of each type among the procedural, conceptual, and applicational types derived from the analysis of Step 3 were listed for each book in an Excel document and were then compared among all eight books. At the same time, the different task sets were added up. A task set refers to a group of tasks for a single type of tasks within a topic. Accordingly, there are procedural sets, conceptual sets and applicational sets. A topic may consist of one, two, or all three types of task sets. A task set may contain a large 
number of the same type of tasks but sometimes may not. A sum and comparison of different types of task sets among the eight books can help in determining what learning opportunities a textbook provides and how widely different task sets spread out within a whole HLT. The three computations in each book were: the total number of task sets in a whole HLT of each book; the total number of all three types of task sets; and the total number of task sets with merely conceptual tasks. The results were generalized in an Excel document. HLT-tasks as the fourth type were listed separately as another category since it consists of a whole sequence of tasks containing the other three types which have already been counted in task sets earlier. Therefore, HLT-tasks were not included in the Excel document.

The basic structure of all the analytical steps is bottom-up. The repeated procedures, from the first two separate dimensions to the summed analysis, strengthen the reliability.

\section{Results}

This analysis has been conducted from both horizontal and vertical dimensions. The report of the results is presented according to these two dimensions. Research Question 1 regarding HLT from a teaching perspective is answered in 5.1, while Research Question 2 regarding learning tasks in every HLT from a learning perspective is answered in 5.2. Two tables (Tables 1-2), two diagrams (Figures 8-9) and six figures (Figures 2-7) are used in order to illustrate the main results. The eight textbooks are referred to as B1-B8 (Appendix).

\subsection{Results in the horizontal dimension}

Results show that embedded HLTs involve in three, four, or five learning processes (Table 1). All eight books present the content concerning quadratic equations primarily in four parts in horizontal order: 1) concepts of polynomials, algebra expressions, or algebra rules; 2) distributive property, multiplication of two different binomials, perfect square rules over addition and subtraction, difference of squares, factoring; 3) solving quadratic equations with the square root method, factoring, completing the square, the $p q$ formula or the graphical method; and 4) the application of quadratic equations in solving real-world problems, or relationships between 
parameters $^{8}$ and roots of quadratic equations or discriminants 9 .

All the books contain the same parts of HLT learning processes (Processes 2 and 3) concerning learning algebra identities and rules as well as solving quadratic equations in different methods, but they differ in Processes 1 and 4 as well as 5. Five of them (B1, B3, B4, B5, B7) consist of four processes, two of them (B2, B6) three processes, and one of them (B8) five processes. The HLT learning processes are the same for Processes 2 and 3. B1 does not include factoring; B3, B4, and B6 contain the most content topics in Process 2.

Table 1. A summary of five learning processes including visual representations used in examples in analysis of horizontal dimension

\begin{tabular}{|c|c|c|c|c|c|}
\hline Books/Processes & 1 & 2 & 3 & 4 & 5 \\
\hline B1 & $\begin{array}{l}\text { Polynomials } \\
\text { GR (DP/MTB) }\end{array}$ & $\begin{array}{l}\text { Algebra rules } \\
\text { GR (DSR) }\end{array}$ & $\mathrm{M} 1,3,4$ & $\begin{array}{l}\text { Algebra } \\
\text { history } \\
\text { Application }\end{array}$ & \\
\hline B2 & $\begin{array}{l}\text { Simplifying } A E \text {, } \\
\text { linear } \\
\text { expressions } \\
\text { Solving linear } \\
\text { equations and } \\
\text { inequalities } \\
\text { Showing results } \\
\text { on number } \\
\text { lines }\end{array}$ & $\begin{array}{l}\text { Algebra rules } \\
\text { Factoring } \\
\text { GR (MTB) }\end{array}$ & M1-4 & & \\
\hline B3 & $\begin{array}{l}\text { Polynomials } \\
\text { Simplifying AE } \\
\text { Solving linear } \\
\text { equations }\end{array}$ & $\begin{array}{l}\text { Algebra rules } \\
\text { Factoring } \\
\text { Solving QES } \\
\text { With DSR PSR }\end{array}$ & $\begin{array}{l}\text { Introduction } \\
\text { of QEs } \\
\text { M1-4 } \\
\text { (CS) }\end{array}$ & $\begin{array}{l}\text { Relationship } \\
\text { between } p, q \\
\text { and roots }\end{array}$ & \\
\hline B4 & $\begin{array}{l}\text { Tasks GR } \\
\text { (DP/MTB/PSR+) } \\
\text { Polynomials }\end{array}$ & $\begin{array}{l}\text { Commutative } \\
\text { Associative } \\
\text { Algebra rules } \\
\text { GR (DP/MTB) }\end{array}$ & $\begin{array}{l}\text { M1-4 } \\
\text { Algebra } \\
\text { history } \\
\text { GR (CS) }\end{array}$ & Application & \\
\hline
\end{tabular}

${ }^{8}$ Parameters of quadratic equations refer to $a, b$, and $c$ in the general quadratic equation $a x^{2}+b x+c=0$ where $a$ and $b$ are coefficients while $c$ is constant.

${ }^{9}$ Discriminant is an expression, $\sqrt{\left(\frac{p}{2}\right)^{2}-q}$ as part of a $p q$ formula. It is used to justify which types of solutions a common quadratic equation can have. A quadratic equation has one real solution when $\left(\frac{p}{2}\right)^{2}-q=0$; two real solutions when $\left(\frac{\mathrm{p}}{2}\right)^{2}-\mathrm{q}>0$; and no real solutions (or solutions with complex numbers) when $\left(\frac{\mathrm{p}}{2}\right)^{2}-\mathrm{q}<0$. 


\begin{tabular}{|c|c|c|c|c|c|}
\hline & & $\begin{array}{l}\text { Factoring } \\
\text { Solving QES } \\
\text { with DSR PSR } \\
\text { (DP/PSR) }\end{array}$ & & & \\
\hline B5 & $\begin{array}{l}\text { Tasks GR } \\
\text { (DP/MTB/PSR+) } \\
\text { Polynomials }\end{array}$ & $\begin{array}{l}\text { Algebra rules } \\
\text { GR of } \\
\text { DP/MTB/PSR- } \\
\text { Factoring } \\
\text { Solving QEs } \\
\text { with DSR PSR } \\
\text { (DP/PSR+) } \\
\end{array}$ & $\begin{array}{l}\text { M1-4 } \\
\text { GR (CS) }\end{array}$ & $\begin{array}{l}\text { Application } \\
\text { Algebra } \\
\text { history }\end{array}$ & \\
\hline B6 & & $\begin{array}{l}\text { Algebra } \\
\text { identity } \\
\text { Commutative } \\
\text { Associative } \\
\text { Algebra rules } \\
\text { GR (MTB) } \\
\text { Factoring } \\
\text { Mixed ex } \\
\text { (DSR/PSR-) }\end{array}$ & $\begin{array}{l}\text { M1-4 } \\
\text { GR (CS) } \\
\text { (CS) }\end{array}$ & $\begin{array}{l}\text { Relationship } \\
\text { between } p, q \\
\text { and roots }\end{array}$ & \\
\hline B7 & $\begin{array}{l}\text { Tasks } \\
\text { QF-gr } \\
\text { (3 types of } \\
\text { roots) }\end{array}$ & $\begin{array}{l}\text { Algebra rules } \\
\text { Factoring } \\
\text { GR(MTB/PSR+) } \\
\text { (PSR-) }\end{array}$ & $\begin{array}{l}\text { QF-gr } \\
\text { (intro QE) } \\
\text { M1-4+M5 } \\
\text { Solve QEs in } \\
\text { complex nr } \\
\text { GR (CS) }\end{array}$ & $\begin{array}{l}\text { Relationship } \\
\text { between } p, q \\
\text { and roots } \\
\text { Discriminants } \\
\text { Tasks QF-gr }\end{array}$ & \\
\hline B8 & $\begin{array}{l}\text { Complex nr } \\
\text { DP } \\
\text { Tasks GR (DP) } \\
\text { Simplifying } \\
\text { linear } \\
\text { expressions } \\
\text { Solving linear } \\
\text { equations }\end{array}$ & $\begin{array}{l}\text { Polynomials } \\
\text { Algebra rules } \\
\text { GR (MTB) } \\
\text { GR (PSR+) }\end{array}$ & $\begin{array}{l}\text { The concept } \\
\text { of QEs } \\
\text { M1, 3, } 4 \\
\text { GR (CS) } \\
\text { (DSR) }\end{array}$ & $\begin{array}{l}\text { Discriminants } \\
\text { Concept of } \\
\text { complex } \mathrm{nr} \\
\text { Complex } \mathrm{nr} \\
\text { as roots to a } \\
\mathrm{QE} \\
\text { Root } \\
\text { equation } \\
\text { Application }\end{array}$ & $\begin{array}{l}\text { Factoring } \\
\text { Solving QEs } \\
\text { with } \\
\text { factoring } \\
\text { M2 }\end{array}$ \\
\hline
\end{tabular}

Note. $A E$ refers to algebra expressions.

$C S$ refers to a solution method for solving quadratic equations and is called completing the square.

$D P$ refers to algebra identity: distributive property $a(b+c)=a b+a c$.

$D S R$ refers to the difference of two square $a^{2}-b^{2}=(a+b)(a-b)$.

$G R$ refers to geometrical representations consisting of combinations of areas of squares and rectangles.

M1-4 refers to four solution methods used for solving quadratic equations: the square root as M1, factoring as M2, completing the square as $\mathrm{M} 3$, and the $p q$ formula as M4.

$P S R \pm$ is a short term for two algebra identities called perfect square rules over addition and subtraction $a^{2} \pm 2 a b+$ $b^{2}=(a \pm b)^{2}$.

$p$ refers to a coefficient of $x$ in the quadratic equation $x^{2}+p x+q=0$. 
$q$ refers to a constant in the quadratic equation $x^{2}+p x+q=0$.

$Q E$ refers to a quadratic equation.

$Q F-g r$ as M5 refers to the approach of solving quadratic equations through searching the $x$-intercepts of a quadratic function graph.

Among the analyzed books, it is also found that visual representations are frequently applied in Processes 2 and 3 for illustrating distributive property (Figure 2), multiplication of two different binomials (Figure 3), perfect square rule over addition (Figure 4), and completing the square (Figures 5-6), which are often presented with geometrical representations for the sums of rectangles and squares. Another type of visual representation illustrates three types of solutions to quadratic equations, represented by three $x$-intercepts of graphs of quadratic functions (Figure 7).

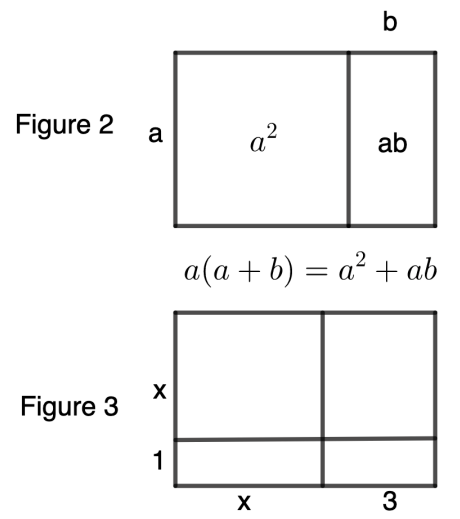

$$
(x+1)(x+3)=x^{2}+4 x+3
$$

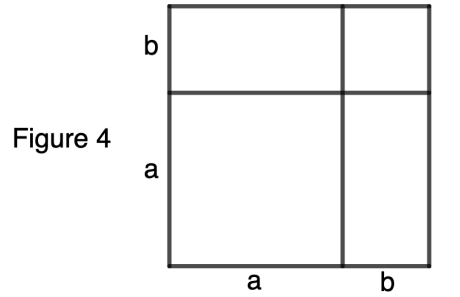

$(a+b)^{2}=a^{2}+2 a b+b^{2}$

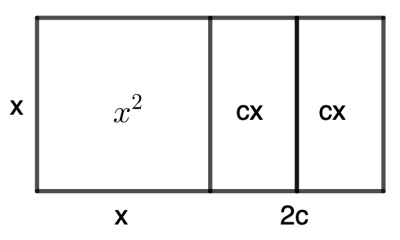

Figure 5

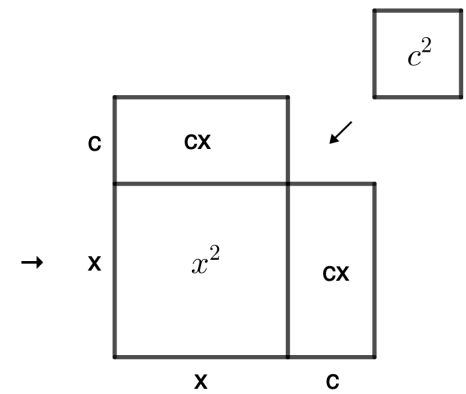

Figure 6

$$
x^{2}+2 c x+?=(x+c)^{2}
$$

Figures 2-6: Geometrical representations of algebra rules and completing the square method (Alfredsson et al., 2011). 


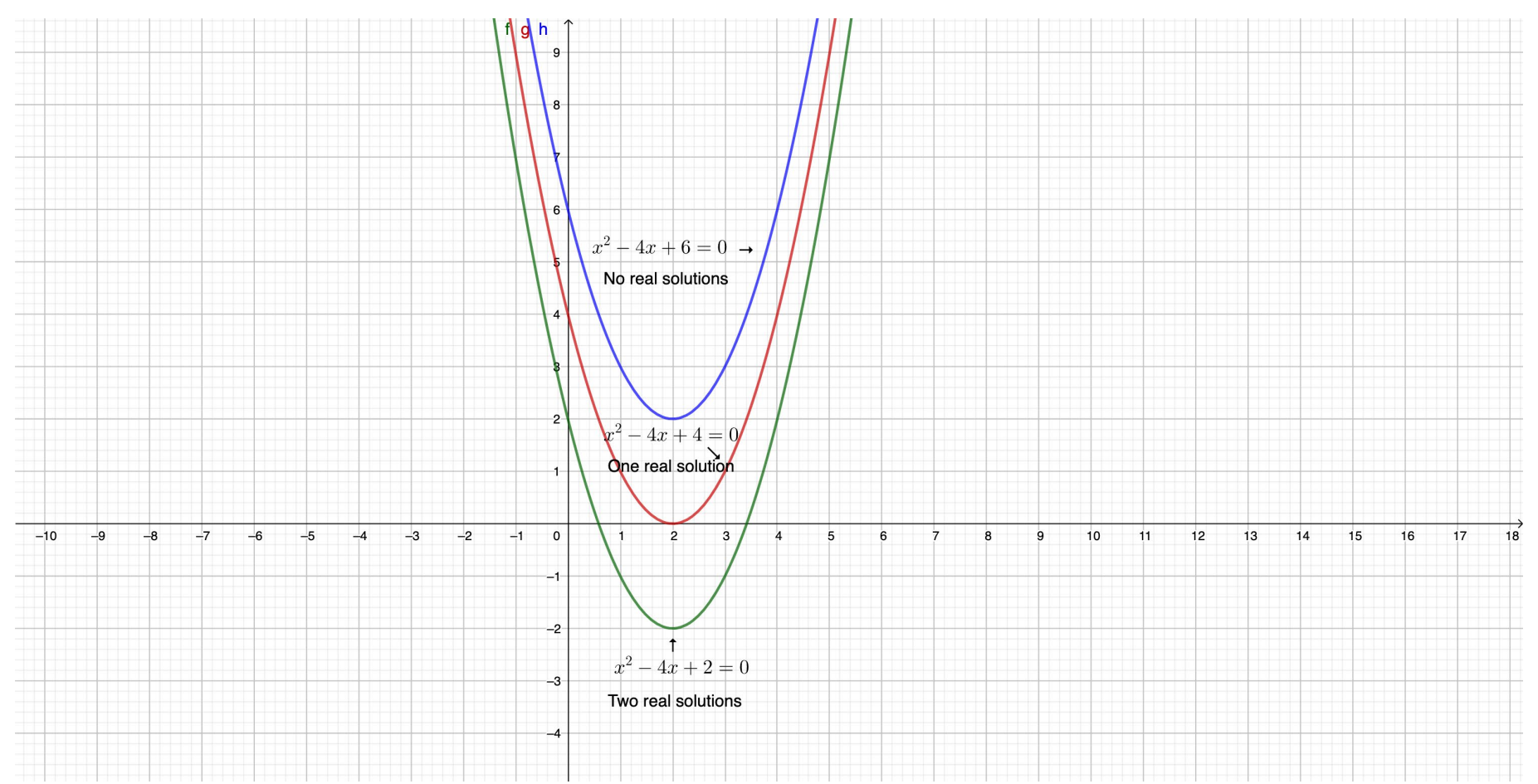

Figure 7. Three types of graphical representations of solutions to quadratic equations, represented by graphs of quadratic functions (Szabo et al., 2012).

Every book illustrates the multiplication of two binomials with geometrical representations as GR. Distributive property in B1, B3, B4, B5 and B6, the difference of two squares in B1, perfect square rule over addition in B5, B7 and B8 are illustrated with GR in Process 2. Solving quadratic equations with completing the square method in B4-8 are illustrated with GR in Process 3. Graphical representations are used in Process 3 twice in B7 but not in the other books. GRs in Process 3 are a combination of early usage of GRs in Process 2 or even Process 1, as in the cases in Figures 2-6 in B4-8, which reflects the development of an HLT. At the same time, graphical representations illustrating three different roots to quadratic equations, as in Figure 7, are applied together with GRs in B7. The combination of GRs is developed from a single figure with rectangles to three figures with squares and rectangles, with the intent of visually transforming how distributive property, multiplication of binomials, and perfect square rules are operated, and then how they are developed into a complex procedure; that is, completing a square as an approach to the $p q$ formula. The emphasis on teaching concepts with the help of visual representations is explored among these five books (B4-8).

Process 3 contains content on solving quadratic equations with three, four, or five different methods: M1, M2, M3, M4, and M5 (Table 1). All the textbooks except B1 present M1-M4. Factoring method is not available in B1. In this process, M3 is 
illustrated with GR (Figures 5-6) as examples in five books (B4-8) just before the introduction of $\mathrm{M}_{4}$, while using quadratic function graphs to solve quadratic equations as M5 is applied in B7 (Table 1). M2 is used for solving particular quadratic equations, which can be only factorized by using algebra identities inversely. Using factoring based on Viete's formula to solve quadratic equations is not found. Processes 2 and 3 are the core learning processes of the whole HLT in every book.

Differences mainly remain in Processes 1 and 4. Process 1 is the beginning of a whole HLT in all the books except B6 (Table 1). Process 1 starts with an introduction of concepts involving polynomials in $\mathrm{B} 1, \mathrm{~B} 3, \mathrm{~B} 4$, and $\mathrm{B}_{5}$; simplifying algebra expressions in $\mathrm{B} 2, \mathrm{~B} 3$, and $\mathrm{B} 8$; solving linear equations in $\mathrm{B} 2, \mathrm{~B}_{3}$, and B8; doing tasks with algebra rules in $\mathrm{B}_{4}$ and $\mathrm{B}_{5}$; doing tasks relating to three types of quadratic equation roots in B7; or explaining complex numbers in B8. B2 presents more procedural knowledge in Process 1, consisting of simplifying algebra expressions, solving linear equations and inequalities, and drawing results on number lines. This beginning takes a long path in Process 1 before arriving at Process 2. It also implies an emphasis on procedural knowledge in the whole HLT in B2. B4 and B5, as the same series, have the same Process 1, providing the same tasks on understanding distributive property, multiplication of two binomials, and perfect square rule over addition with geometrical representations, before Process 2 presents these algebra rules in detail. B3 starts Process 1 with both polynomial concepts and operational procedures: simplifying algebra expressions and solving linear equations. B8 contains most of the algebra topics in Process 1 - history of complex numbers, distributive property, simplifying algebra expressions, and solving linear equations - although it introduces polynomial concepts at the beginning of Process 2. Like B2, B8 has a long Process 1 before beginning Process 2. B6 starts its HLT differently from the other books, starting with concepts of algebra identities in Process 2 instead, which means that it contains a shorter HLT, consisting of three learning processes.

Processes 4 and 5 show how an HLT ends. There are three types of endings (Table 1): ending with application of quadratic equations (QEs) to solve real-world problems and the presentation of algebra history related to solving equations to different degrees in $\mathrm{B}_{1}, \mathrm{~B}_{4}$, and $\mathrm{B}_{5}$; ending with presenting the relationship between a coefficient, a constant, and roots of QEs in B3, B6, and B7, among which B7 includes finding the type of solution by analyzing the discriminant of a $\mathrm{QE}$; ending with solving QEs using four methods in Process 3 in B2 and using factoring method in Process 5 
in B8. This results in $\mathrm{B} 2$ having a shorter HLT like B6, while B8 having the longest HLT.

The endings with the application of QEs in B1, B4, and B5 show that these three books emphasize the use of QEs in real-world problems. The endings in the three books $\mathrm{B} 3, \mathrm{~B} 6$, and $\mathrm{B} 7$ presenting discriminants and the relationship between coefficient, constant, and roots lead to an encouragement of more conceptual learning with a focus on mathematics theory.

The differences in Processes 1 and 4 provide different lengths of HLTs among all the books, depending on the number of content topics presented in these two processes. The increase in content topics in $\mathrm{B}_{5}-8$ is a result of adopting the new curriculum. For example, complex numbers are not presented in $\mathrm{B} 2$ but are presented in detail in B7 and B8. Root equations are also included in B8. Thus, B8 provides the longest HLT with the most content topics among all the textbooks. B1 has missed factoring as a solution method in its whole HLT. B3 and B6, as the same series, provide the shortest HLTs in three processes but do not have fewer content topics, which implies that their HLTs are effective.

\subsection{Results in the vertical dimension}

Tasks provided in every book were analyzed according to four types - procedural, conceptual, and applicational as well as HLT-tasks - in each HLT learning process. The result of analyzing tasks according to the first three types shows that some processes consist of all the first three types of tasks, while others do not. In each HLT, there are more procedural tasks than conceptual and applicational ones though the sum of conceptual and applicational tasks surpasses procedural tasks in B3-7 (Figure 8). Among the eight books, B6 provides the most numbers of conceptual and applicational tasks, while B8 provides the most numbers of procedural tasks. Every topic contains a number of task sets. B3 (Figure 9) provides the most sets (13) as well as the most PCA sets ${ }^{10}(8)$, while B4, B7 and B8 provide the most conceptual sets (11). The higher number of task sets indicates that tasks are widely spread in a whole HLT but does not necessarily increase the number of tasks. B3 has less numbers of tasks than $\mathrm{B} 8$ but distributes the tasks among a wide range of topics in the whole HLT. With the most PCA sets, B3 emphasizes on developing all the three types of mathematics practices: procedural, conceptual and applicational.

\footnotetext{
${ }^{10}$ PCA sets refer to task sets consisting of all three types of tasks: procedural, conceptual, and applicational.
} 


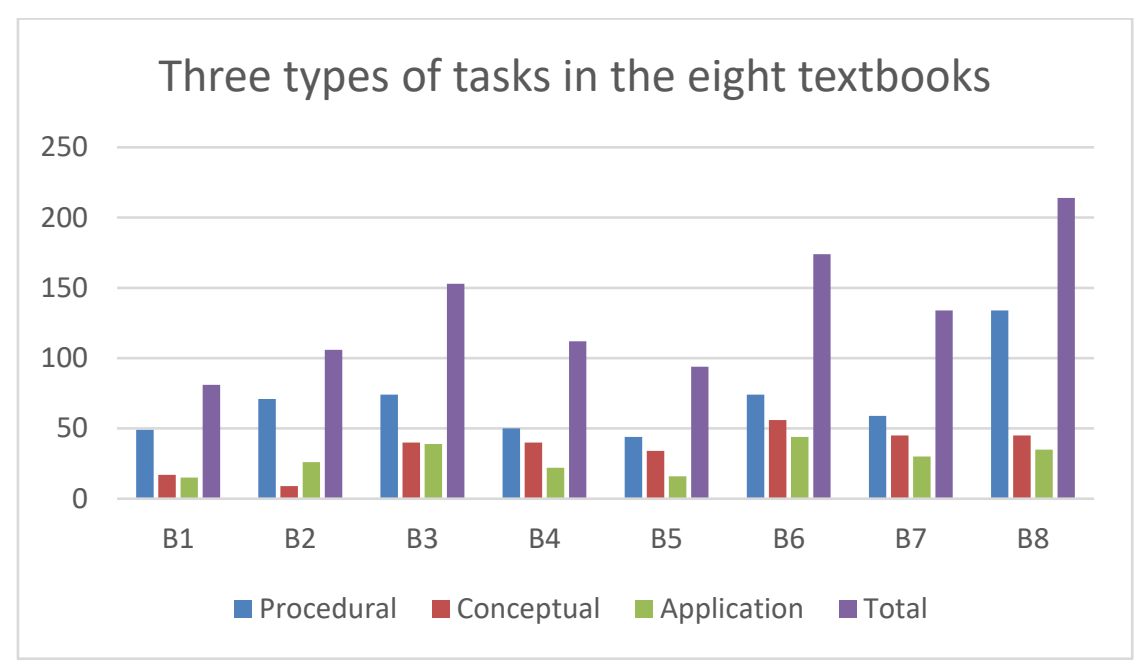

Figure 8. Three types of tasks in the eight textbooks.

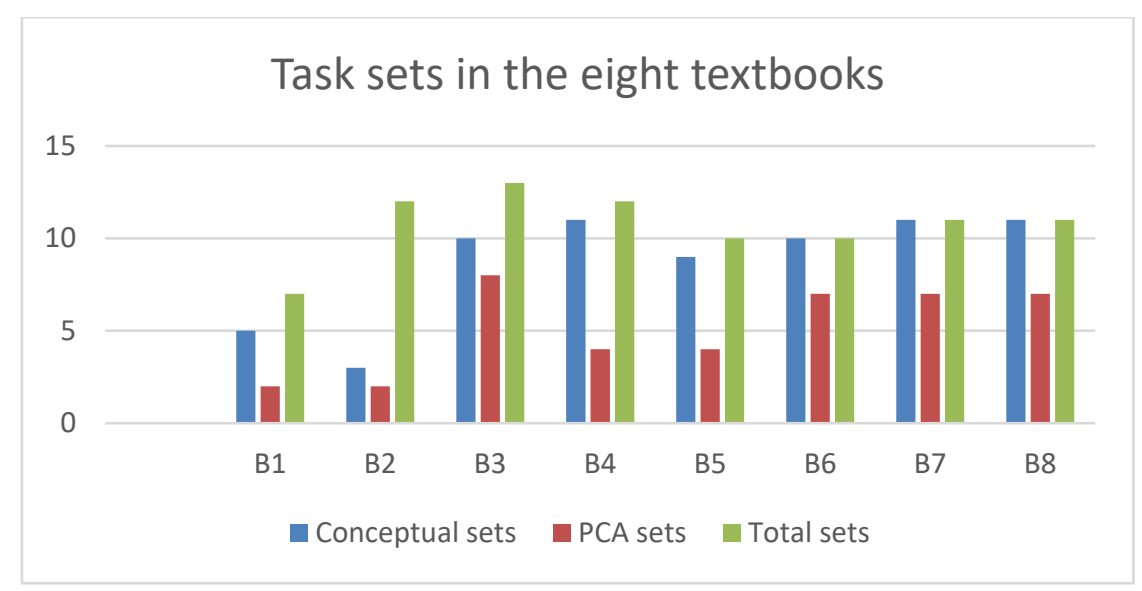

Figure 9. Task sets in the eight textbooks.

The mathematics procedures among the procedural tasks mainly involve simplifying, computing, or factoring algebra expressions; solving linear equations; solving linear inequalities; or solving quadratic equations using four different methods. Most of the quadratic equations in all the books contain integer parameters, besides a few cases with fractions or decimal numbers. The majority of the quadratic equations can be solved using both factoring and the $p q$ formula. B7 presents one more solution method entailing solving QEs graphically using calculators. There is not much variation in procedural tasks among the books. The procedures for doing these tasks can be found and imitated in previously provided examples, which means that they are routine tasks (Brändström, 2005). Four of the eight books - B2, B3, B6, and B8 - provide mainly procedural tasks, with B8 providing the most. B1, B2, and B8 provide unbalanced learning opportunities, with much more procedural tasks than 
conceptual and applicational ones, while B6 and B7 gave a balanced task distribution (Figure 8). B3 and B6 provide tasks containing advanced procedures.

The common types of conceptual tasks (Table 2) are: finding a constant, a coefficient or an unknown $x$ in an equation (B1, B3, B5-8); finding or analyzing the relationship between a coefficient, a constant, and roots (B1, B3-8); proving or explaining algebra identities such as the difference of two squares, perfect square rules over addition and subtraction, or the method for solving quadratic equations called Completing the Square - with geometrical representations (B1, B3-8), especially in B7; assessing whether or justifying that calculation procedures or solutions or the equivalence of a QE are correct (B1, B3-7); reasoning why a solution or calculation procedure is wrong (B4-5, B7-8); and setting up algebra expressions (B2, B5, B8). There are also other types, such as: drawing a number line to illustrate solutions (B2); matching up graphs of quadratic functions with their QE solutions and discerning between complex numbers and real numbers (B7); and discussing an equation with no solution (B8).

B6, B7, and B8 have more conceptual tasks than the other five books, while B6 provides the most conceptual tasks (Figure 8). At the same time, B5, B7 and B8 provide more varying conceptual tasks than the other five books (Table 2). The rich conceptual tasks in B6 and B7-8 demonstrate the emphasis on conceptual learning in these books. B2 provides the least conceptual tasks.

Table 2. Different types of conceptual tasks in every textbook in the vertical dimension analysis

\begin{tabular}{cl}
\hline Textbooks & Different types of conceptual tasks \\
\hline B1 & $\begin{array}{l}\text { Assess, find constant and variable, find relationship between } p, q \text {, and roots, prove } \\
\text { equivalence, prove PSR } \pm \text { with GR }\end{array}$ \\
\hline B2 & Set up algebra expressions, draw number lines to show solutions \\
\hline B3 & $\begin{array}{l}\text { Describe, compare, identify, analyze, assess, find constant and variable, investigate } \\
\text { relationship between } p, q, \text { and roots, explain CS with GR }\end{array}$ \\
\hline B4 & $\begin{array}{l}\text { Discover, interpret, reason, compute, generalize, assess, analyze relationship between } p, q, \\
\text { and roots, explain MTB, PSR+ with GR }\end{array}$ \\
\hline B5 & $\begin{array}{l}\text { Give examples, set up, prove, reason, compute, generalize, assess, find constant and } \\
\text { variable, analyze relationship between } p, q \text {, and roots, explain DP, PSR+ with GR }\end{array}$ \\
\hline B6 & $\begin{array}{l}\text { Assess, find constant and variable, find relationship between } p, q, \text { and roots, prove DSR, } \\
\text { PSR- with GR (advanced) }\end{array}$ \\
\hline Describe, discern, match up, prove with the Pythagorean Theorem, reason, assess, find \\
constant and coefficient, find relationship between $p, q$, and roots, prove \& explain PSR-, CS \\
with GR
\end{tabular}


Like the other two task types, applicational tasks are distributed in different learning processes of a whole HLT in every book. Seven books (B1, B3-8) provide fewer applicational tasks than procedural and conceptual ones, while B2 provides more applicational tasks than conceptual ones (Figure 8). B1 provides the least, and B6 the most, applicational tasks. Most of the applicational tasks involve solving a textual problem by setting up an algebra expression or a quadratic equation or function according to a given description and then computing some specific value; interpreting a given mathematics model such as an algebra expression, a quadratic function or equation and then calculating some specific value; or reasoning solutions. They are related to three or four topics: arithmetic (finding number sequences, even or odd numbers), economy (saving money or shopping), geometry (areas, sides, or circumferences of rectangles, squares, circles, or triangles), or physics (height of a throwing object, driving distances). A few problems are challenging and cannot be helped with previous examples or theoretical presentations, but rather require creative or high mathematical thinking. Some of them are not necessarily difficult but require thinking from other perspectives, but this type of problem is rare.

The result of analyzing the fourth type of tasks shows that only $\mathrm{B} 4$ and $\mathrm{B} 5$ provide HLT-tasks with requirements: computing (B4, B5), discovering (B4), generalizing (B4, B5), and interpreting (B4). These requirements, found in seven sequences of tasks in B4 and B5, are identified as HLT sequences, meaning that every sequence of tasks is structured from basic computing procedures to find relationships between computations and results, so that students are required to generalize abstract algebraic rules. These requirements encourage investigating and discovering activities that are more complex than other tasks, and require high-level thinking and mathematical competencies (Gracin, 2018; Niss, 2015).

Briefly, the vertical results show that B3 spreads out more exercises among a wide range of topics and includes all the first three types of the tasks most of the time in the whole HLT. B1 provides the least of tasks, while B8 provides the most of tasks. B6 encourages both conceptual learning and mathematics applications, while B8 encourages both procedural and conceptual learning. B4 and B5 encourage mathematics thinking according to an HLT progression.

To conclude the results of the analyses in both dimensions, all the embedded HLTs are similar for the presentation of core content, which is algebra identities and rules and quadratic equations. The eight analyzed textbooks are therefore similar. But they are different when it comes to how an HLT starts and ends as well as what types of 
tasks are provided. B1, B4, and B5 start with introducing polynomials and end with applying quadratic equations to solve real-world problems, while $\mathrm{B} 4$ and $\mathrm{B} 5$ are more similar since they introduce algebra rules by presenting tasks with GRs. B1 provides the least tasks. B2, B3, and B8 have similar starts with procedures of simplifying expressions and solving linear equations. B6 starts its HLT directly with algebra rules at Process 2 without Process 1. B7 starts with tasks related to three types of roots represented by graphical representations. B3, B6, and B7 end their HLTs by finding relationships between roots and constants, including coefficients of quadratic equations, while B2 and B8 end their HLTs by solution methods to quadratic equations. B8 has the longest HLT since it covers the most topics and provides the most tasks involving the most procedural tasks, while B2 and B6 have the shortest HLTs though B6 is more effective because of the most numbers of conceptual and applicational tasks within its HLT. B7 applies the most visual representations within its HLT. The application of geometrical representations in B4-8 visually shows the development of algebra rules within their HLTs. B4 and B5 provide similar HLTtasks. B3 and B6 provide advanced tasks comparing to the other books. Procedural tasks are more than conceptual and applicational ones in each book but the sum of the last two types is more than procedural ones in B3-7.

\section{Discussion}

The results have shown similar HLTs concerning the core contents on algebra rules and quadratic equations among the eight Swedish textbooks. But they are different when it comes to how an HLT starts and ends. The explored pedagogy implies that teaching in basic algebra concepts and procedural skills is regarded as the essential pre-knowledge before teaching quadratic equations; illustrating related algebra rules or solutions methods of quadratic equations with geometrical representations is a pedagogical approach to abstract algebra and intends to develop students' conceptual understanding. Enriched through two types of visual representations, the content in B7 can be understood easily. Thus, B7 emphasizes conceptual learning. The Swedish textbooks' application of geometrical representations or geometrical models (Pieronkiewicz \& Tanton, 2019) for completing the square is related to algebra history (e.g., Katz \& Barton, 2007) and shows its historical aspect. The different endings of the HLTs indicate two types of intended pedagogy: learning for mastering mathematics theory, and learning for applications. A long HLT with many topics but a large number of procedural tasks in B8 implies teaching basic concepts and 
procedures. A short HLT in B6 without a focus on pre-knowledge implies an effective learning process for advanced learners.

The main learning opportunity provided by the textbooks is the practice of procedural tasks. This implies that teaching algebra procedures is a basic pedagogical approach. On the other hand, conceptual and applicational learning is encouraged in B3-7 especially in B6. Various conceptual tasks and the HLT-tasks in B4 and B5 intend to provide students opportunities to develop their mathematics thinking within conceptual learning progression (Simon \& Tzur, 2004). Similar to algebra big ideas (Hemmi et al., 2018), this study finds categories of equivalence, expressions, equations, inequalities, variables, generalized arithmetic, function thinking in the analyzed tasks. Among these categories, generalized arithmetic and function thinking as well as inverse properties are frequently represented in the tasks. The results of the analyses in both dimensions also show an agreement with the five algebra discourses in the previous study by Palm Kaplan (2019). Geometrical, realistic and scientific discourses are common among the eight textbooks.

None of the Swedish textbooks uses graphical representations to illustrate the $p q$ formula, as suggested by Edwards and Chelst (2019) with the purpose of helping students understand the abstract quadratic formula. The quadratic formula is not presented as the most common method for solving quadratic equations in the Swedish textbooks; while the $p q$ formula is presented as the most common approach, it is a simplification of the quadratic formula. This result agrees with Olteanu and Holmqvist's (2012) in their study. The application of geometrical representations for completing the square intends to transform the geometrical approach to the abstract $p q$ formula among five books (B4-8). Teaching for conceptual learning is explored. The use of the factoring method to solve quadratic equations based on Viete's formula or the AC method is not found. Therefore, the factoring method among all the textbooks is utilized limitedly for solving simple quadratic equations. The reason for this may be that quadratic equations of the type $a x^{2}+b x+c=0$ are not directly presented in any of the books, even though this type of equation appears in some tasks and has to be handled by first dividing by the coefficient of $a$. Consequently, the quadratic formula is not presented in the books.

This study's two-dimensional analyses with the HLT framework (Simon, 1995, 2004) and conceptual as well as procedural knowledge (Hiebert \& Lefevre, 1986) have allowed for an examination of mathematics content considering both theoretical presentations with examples and representations and tasks provided widely and 
deeply from both the teaching and learning perspectives. Therefore, the research questions have been able to be answered. However, the analysis of tasks found a dilemma when tasks couldn't be clearly discerned as pure conceptual and applicational types since computation is a necessary step to carry out conceptual and applicational steps. In this case, identifying a task type was based on the cognitive demands of a task. In future studies, it could be interesting to examine how a specific algebra concept, rule, or solution method can be understood by students using one of the textbooks analyzed here.

\section{Acknowledgements}

I wish to express my great appreciation to my supervisors, as well as my fellow researchers and the group of doctoral students who read and contributed their valuable opinions to this study and this paper.

\section{References}

Allaire, P. R., \& Bradley, R. E. (2001). Geometric approaches to quadratic equations from other times and places. Mathematics Teacher, 94(4), 308-319.

Bergwall, A., \& Hemmi, K. (2017). The state of proof in Finnish and Swedish mathematics textbooks - capturing differences in approaches to upper-secondary integral calculus. Mathematical Thinking and Learning, 19(1), 1-18. Doi: https://doi.org/10.1080/10986065.2017.1258615

Bossé, M. J., \& Nandakumar, N. R. (2005). The factorability of quadratics: Motivation for more techniques. Teaching Mathematics and its Applications: An International Journal of the IMA, 24(4), 143-153.

Brändström, A. (2005). Differentiated tasks in mathematics textbooks: Analysis of the levels of difficulty. (Doctral thesis). Luleå: Luleå tekniska universitet.

Didis, M. G., \& Erbas, A. K. (2015). Performance and difficulties of students in formulating and solving quadratic equations with one unknown. Educational Sciences: Theory \& Practice, 15(4), 1137-1150.

Edwards, T. G., \& Chelst, K. R. (2019). Finding meaning in the quadratic formula. The Mathematics Teacher, 112(4), 258-260. Doi: https://doi.org/10.5951/mathteacher.112.4.0258

Fachrudin, A. D., Putri, R. I. I., \& D. (2014). Building students' understanding of quadratic equation concept using naïve geometry. Indonesian Mathematical Society Journal on Mathematics Education, 5(2), 192-202.

Gracin, D. G. (2018). Requirements in mathematics textbooks: a five-dimensional analysis of textbook exercises and examples. International Journal of Mathematical Education in Science and Technology, 49(7), 1003-1024. Doi: https://doi.org/10.1080/0020739x.2018.1431849

Hemmi, K., Bråting, K., Liljekvist, Y., Prytz, J., Madej, L., Pejlare, J., \& Palm Kaplan, K. (2018). Characterizing Swedish school algebra - initial findings from analysis of steering 
documents, textbooks and teachers' discourses. In E. Norén, H. Palmér \& A. Cooke (Eds.), Nordic research in mathematics education. Papers of NORMA 17 (pp. 299-308).

Gothenburg: SMDF.

Hemmi, K., Lepik, M., Madej, L., Bråting, K., \& Smedlund, J. (2019). Introduction to early algebra in Estonia, Finland and Sweden - some distinctive features identified in textbooks for Grades 1-3. In U. T. Jankvist, M. Van den Heuvel-Panuizen \& M. Veldhuis (Eds.), Proceedings of the Eleventh Congress of the European Society for Research in Mathematics Education (CERME11, February 6-1O, 2019) (pp. 2039-2046). Utrecht: Freudenthal Group \& Freudentha Institute, Utrecht University and ERME.

Hiebert, J., \& Lefevre, P. (1986). Conceptual and procedural knowledge in mathematics: An introductory analysis. In J. Hiebert (Ed.), Conceptual and procedural knowledge: The case of mathematics (pp. 1-27). Hillsdale, NJ: Lawrence Erlbaum.

Hong, D. S., \& Choi, K. M. (2014). A comparison of Korean and American secondary school textbooks: the case of quadratic equations. Educational Studies in Mathematics, 85: 241263. Doi: https://doi.org/10.1007/s10649-013-9512-4

Jablonka, E., \& Johansson, M. (2010). Using texts and tasks: Swedish studies on mathematics textbooks. In B. Sriraman, C. Bergsten, S. Goodchild, G. Palsdottir, B. Dahl, B. D. Söndergard \& L. Haapsalo (Eds.), The first sourcebook on Nordic research in mathematics education (pp. 363-372). Charlotte: Information Age Publishing.

Jakobsson-Åhl, T. (2006). Algebra in upper secondary mathematics: a study of a selection of textbooks used in the years 1960 - 2000 in Sweden. (Licential thesis). Luleå: Luleå University of Technology.

Johansson, M. (2006). Teaching mathematics with textbooks: A classroom and curricular perspective. (Doctral thesis). Luleå: Luleå University of Technology.

Johnsen, E. B. (1993). Textbooks in the Kaleidoscope: A critical survey of literature and research on educational texts. Oslo: Scandinavian U. P, cop.

Katz, V., \& Barton, B. (2007). Stages in the history of algebra with implications for teaching. Educational Studies in Mathematics, 66(2), 185-201.

Lepik, M., Grevholm, B. \& Viholainen, A. (2015). Using textbooks in the mathematics classroom the teacher's view. Nordic Studies in Mathematics Education, 2O(3-4). 129-156.

Madej, L. (2021). X-men sen då? Algebrans stora idéer från första klass till högre matematik. Med focus på tidig algebra i Sverige. (Doctoral thesis). Department of Education, Uppsala University.

Niss, M. (2015). Mathematical competencies and PISA. In K. Stacey \& R. Turner (Eds.), Assessing Mathematical Literacy (pp. 35-55). Dordrecht: Springer.

Olteanu, C., \& Holmqvist, M. (2012). Differences in success in solving second-degree equations due to the differences in classroom instruction. International Journal of Mathematical Education in Science and Technology, 43(5), 575-587.

Palm Kaplan, K. (2019). International large-scale assessments and mathematics textbooks in a curriculum reform process. Changes in lower secondary school algebra in Sweden 19952015. (Doctoral thesis). Department of Education, Uppsala University.

Palm, T., Boesen, J., \& Lithner, J. (2011). Mathematical reasoning requirements in Swedish upper secondary level assessments, Mathematical Thinking and Learning, 13(3), 221-246, Doi: https://doi.org/10.1080/10986065.2011.564994

Pepin, B., Haggarty, L., \& Keynes, M. (2001). Mathematics textbooks and their use in English, French and German classrooms: A way to understand teaching and learning cultures. Zentralblatt für Didaktik der Mathematik, 33(5), 158-175. 
Pieronkiewicz, B., \& Tanton, J. (2019). Different ways of solving quadratic equations. Annales Universitatis Paedagogicae Cracoviensis, 11 (2019), 103-125. Doi:

https://doi.org/10.24917/20809751.11.6

Saglam, R., \& Alacaci, C. (2012). A comparative analysis of quadratics unit in Singaporean, Turkish and IBDP mathematics textbooks. Turkish Journal of Computer and Mathematics Education, 3(3), 131-147.

Simon, M. (2014). Hypothetical learning trajectories in mathematical education. In S. Lerman (Ed.), Encyclopedia of mathematics education (pp. 272-275). Springer Netherlands. Doi: https://doi.org/10.1007/978-94-007-4978-8

Simon, M. A. (1995). Reconstructing mathematics pedagogy from a constructivist perspective. Journal for Research in Mathematics Education, 26(2), 114-45.

Simon, M. A., Kara, M., Placa, N., \& Avitzur, A. (2018). Towards an integrated theory of mathematics conceptual learning and instructional design: The Learning Through Activity theoretical framework. Simon, M. A., Journal of Mathematical Behavior (2018), https://doi.org/10.1016/j.jmathb.2018.04.002

Simon, M. A. \& Tzur, R. (2004). Explicating the role of mathematical tasks in conceptual learning: An elaboration of hypothetical learning trajectory. Mathematical Thinking and Learning, 6(2), 9-104. Doi: https://doi.org/10.1207/s15327833mtlo602_2

Skolverket. (2011). Curriculum for the upper secondary school: subject of mathematics. Retrieved July 3, 2020 from https://www.skolverket.se/undervisning/gymnasieskolan/laroplanprogram-och-amnen-i-gymnasieskolan/hitta-program-amnen-och-kurser-igymnasieskolan

Vaiyavutjamai, P., \& Clements, M. A. (2006). Effects of classroom instruction on students' understanding of quadratic equations. Mathematics Education Research Journal, 18(1), 47.

Valverde, G. A., Bianchi, L. J., Wolfe, R. G., Schmiddt, W. H., \& Houang, R. T. (Eds.). (2002). According to the book: Using TIMSS to investigate the translation of policy into practice through the world of textbooks. Dordrecht: Kluwer Academic Publishers.

Winsløw, C. (2004). Quadratics in Japanese. Nordic Studies in Mathematics Education, 9(1), 5174 . 


\section{Appendix: List of mathematics textbooks included in this study}

Alfredsson, L., Bråting, H., Erixon, P., \& Heikne, H. (2011). Matematik 5000 2C. Stockholm: Natur \& Kultur.

Alfredsson, L., Brolin, H., Erixon, P., Heikne, H., \& Ristamäki, A. (2007). Matematik 4000 B (Blå). Stockholm: Natur \& Kultur.

Björup, K., Körner, S., Oscarsson, E., \& Sandhall, Å. (2000). Nya Delta matematik. Kurs A och B. Malmö: Gleerups Utbildning AB. (B2)

Gennow, S., Gustafsson, I-M., \& Silborn, B. (2008). Exponent B (Röd). Malmö: Gleerups Utbildning $\mathrm{AB}$.

Gennow, S., Gustafsson, I-M., \& Silborn, B. (2012). Exponent 2C. Malmö: Gleerups Utbildning AB. Sjunnesson, J., Holmström, M., \& Smedhamre, E. (2011). Matematik 2c. Stockholm: Liber AB.

Szabo, A., Larson, N., Viklund, G., Dufåker, D., \& Marklund, M. (2012). Matematik origo $2 c$. Stockholm: Sanoma Utbildning.

Wallin, H., Lithner, J., Wiklund, S., \& Jacobsson, S. (2000). $\Delta N T / a+b-L i b e r$ Pyramid. Gymnasiematematik för NV och TP, kurs A och B. Stockholm: Liber AB.

A short list in the form of numbers:

B1: $\Delta \mathrm{NT} / \mathrm{a}+\mathrm{b}-$ Liber Pyramid. Gymnasiematematik

B2: Nya Delta matematik. Kurs A och B

B3: Exponent B (Röd)

B4: Matematik 4000 B (Blå)

B5: Matematik 5000 2C

B6: Exponent 2C

B7: Matematik origo 2C

B8: Matematik 2c 